\title{
UTILIZACIÓN DEL MODELO DE ESQUEMAS COGNITIVOS DE BASE (SCB) PARA LA CONFIRMACIÓN DEL NÚCLEO DE UNA REPRESENTACIÓN SOCIAL. ANÁLISIS DEL MOVIMIENTO ANTIGLOBALIZACIÓN
}

\author{
Manuel Cárdenas \\ Universidad Católica del Norte \\ Roberto Rodríguez \\ Universidad Complutense de Madrid
}

\begin{abstract}
RESUMEN: En el presente artículo utilizamos el método de confirmación de la centralidad del núcleo de una representación social basado en una adaptación del modelo de esquemas cognitivos de base. Hemos trabajado sobre la base de datos de un estudio previo sobre la representación social del movimiento Antiglobalización. Se pretende determinar si los elementos cuantitativamente más relevantes detectados entonces son, a la vez, aquellos de mayor conectividad (relevancia cualitativa). También se analiza el tipo de conexiones establecidas entre dichos elementos centrales (sobre la base de las cinco familias de esquemas especificadas por la teoría de SCB y sus correspondientes conectores) y los conceptos asociados a dichos estímulos.
\end{abstract}

PALABRAS CLAVE: representaciones sociales; movimiento antiglobalización; esquemas cognitivos de base (SCB).

\section{USING THE MODEL OF BASIC COGNITIVE SCHEMES (SCB) FOR THE CORROBORATION OF THE CENTRAL CORE OF A SOCIAL REPRESENTATION. ANALYZING THE ANTI-GLOBALIZATION MOVEMENT}

\begin{abstract}
In this paper we used a method for the corroboration of the central core of a social representation based on an adaptation of the Model of Basic Cognitive Schemes. We have worked on the data from a previous study on social representation of the Anti-globalization movement. This research seeks to determine if the most relevant quantitative elements are, simultaneously, those of greater connectivity (qualitative relevance). It also seeks to analyze the type of connections established between these central elements (on the base of the five families of schemes specified by the theory of SCB and its corresponding connectors) and the concepts associated to these stimuli.
\end{abstract}

KEYWORDS: social representations; anti-globalization movement; basic cognitive schemes (SCB).

Desde sus primeras formulaciones, la teoría de las representaciones sociales (Moscovici, 1961) no ha dejado de ser progresiva y continuamente afinada. Una de las aportaciones más relevantes y conocidas es la entrada en escena de la llamada "hipótesis del núcleo central" (Abric, 1976, 1989) que en términos generales afirma que la representación se organiza en torno de un núcleo central, al cual proponen distinguir de aquellos elementos de carácter periférico. Dicho núcleo estaría formado por un conjunto reducido de elementos que determinarían la organización y el significado de la representación. De este modo, las representaciones sociales se organizarían en torno de un doble sistema complementario: el sistema central y el sistema periférico (Abric, 1994, 2003).

El sistema central sería más estable y resistente al cambio, hecho que aseguraría la continuidad y permanencia de una representación. Otra característica de este sistema sería su función consensual, en sentido que contendría los elementos de base comunes y compartidos de un grupo. Desde esta perspectiva, el sistema central sería condicionado por la historia grupal y la memoria colectiva, y por ello sería poco sensible a los elementos contextuales más inmediatos.

El sistema periférico dependería, o sería gobernado, directamente por el sistema central (Rouquette, 1994). Su función principal sería concretar el sistema central a través de ciertas conductas determinadas. Permitiría, por otra parte, que las representaciones puedan arraigar en la realidad. Se trataría de un sistema flexible que permitiría al sistema central adaptarse a la realidad concreta en que se encuentra inmerso el grupo portador de la representación. Se trataría de esquemas que prescribirían comportamientos (Flament, 1989) y que tendrían la finalidad de proteger el núcleo de la representación. De este modo, los elementos periféricos podrían mutar flexiblemente de modo de permitir la continuidad y proteger a los elementos centrales.

Desde esta perspectiva, dos representaciones son diferentes únicamente si sus núcleos difieren y serían iguales en tanto los elementos centrales de una y otra no varían, independientemente de que no coincidan sus elementos periféricos. De este modo, puede afirmarse que los elementos periféricos son los que permiten las variaciones indi- 
viduales ligadas a la historia individual de los sujetos (Flament, 1994), de tal manera que lo consensual de la representación se refiere únicamente a los elementos de su núcleo.

La teoría del núcleo ha llegado a precisar dos funciones fundamentales de los elementos del núcleo: "generadora" (los elementos centrales otorgarían sentido a las otras cogniciones de la representación) y "organizadora" (las cogniciones centrales determinarían los lazos que unen las cogniciones periféricas) (Abric, 1994; Moliner, 1989, 1994). Lo anterior implicaría que la centralidad de una representación social no podría ser reportada únicamente por una dimensión cuantitativa (saliencia o grado de conectividad), sino que debe serlo también de forma cualitativa (asociatividad y poder simbólico). Esto es, los elementos centrales tendrían un mayor número de conexiones que aquellos de carácter periférico y serían más fácilmente evocables, pero también definirían la calidad de las conexiones con los restantes elementos.

De este modo, para determinar los elementos centrales de una representación se debería poder dar cuenta no sólo de la importancia cuantitativa, sino, además, mostrar su relevancia cualitativa (sobre todo teniendo en cuenta de que en ocasiones los primeros pueden enmascarar los segundos, o que estos últimos pueden por sobre activación aparecer como más salientes).

Existen disponibles diversos métodos para testar, identificar o confirmar la centralidad de los elementos del núcleo de una representación (Abric, 1994; Guimelli, 1995, 2003; Guimelli \& Rouquette, 1992; Moliner, 1992, 1995; Verges, 1992). Nosotros en particular nos centraremos en el Modelo de Esquemas Cognitivos de Base (SCB) y que tiene como presupuesto que una representación social puede ser considerada como un conjunto de cogniciones, ligadas por lazos de diversa naturaleza pero formalizables por medio de una cantidad finita de "conectores" (Flament, 1994; Flament \& Rouquette, 2003; 2002; Guimelli, 2003; Guimelli \& Rouquette, 1992; Moliner, Rateau \& CohenScali, 2002). Estos conectores (un total de 28 en el modelo actual) se organizarían en cinco familias denominadas esquemas: Léxico (refiere a equivalencias de uso, analogías y significación opuesta), Vecindad (elementos incluyentes, incluidos o a elementos relevantes incluidos con el inductor en otro concepto más general), Oposición (remite a elementos de los que el inductor es componente, a otros que componen al inductor, $\mathrm{y}$ a aquellos que son considerados componentes de un mismo concepto de referencia), Praxis (remite a la acción que designa el inductor, al sujeto sobre la que es aplicada una acción, a una herramienta o estrategia útil para el actor, al autor de la acción, al objeto sobre el que se aplica la acción, a una modalidad particular de acción sobre el objeto, etc.) y atribución (remite a los atributos del concepto - ya sean permanentes, frecuentes u ocasionales -, a atributos normativos, evalua- tivos o causales y a las consecuencias, fines o efectos) y que, en su conjunto, darían cuenta de los elementos estructurales de la representación.

La lógica del modelo SCB consiste en pedir a los sujetos que asocien dos ítems por medio de un conector $u$ "operador de relación" (ítem inductor - OPERADOR Ítem inducido). Dichos operadores remitirían a las familias de conectores, permitiendo obtener un índice de conectividad, que en conjunto con el índice cuantitativo de accesibilidad (palabras más frecuentemente asociadas por los sujetos a un determinado estímulo) nos permitirían identificar sistemáticamente los elementos centrales de una representación social.

Este modelo nos permitirá comprobar si aquellos elementos cuantitativamente relevantes de la representación del Movimiento Antiglobalización (MAG) estudiados en otro artículo (Cárdenas \& Blanco, 2004) poseen además las propiedades cualitativas requeridas para afirmar su centralidad. En este sentido, nos aportarán información sobre los elementos asociativos y semánticos de la representación. Por otra parte, los cinco esquemas referidos nos entregarán información sobre los elementos descriptivos, prescriptivos y atributivos asociados a la RS del movimiento analizado.

\section{Método}

\section{Muestra}

La muestra se compuso de un total de 85 sujetos (estudiantes de primer año de la carrera de psicología en la Universidad Autónoma de Madrid), 32 hombres (38.1\%) y 52 mujeres (61.9\%), con una media de 22.08 años (desviación típica de 1.785), y siendo el rango de edades comprendido entre 18 y 29 años.

\section{Instrumento}

El instrumento analizado consiste en una traducción y adaptación del instrumento utilizado para analizar los SCB (Guimelli, 1994, 2003) y que consta de tres etapas: a) asociación continua. Se presenta a los sujetos un término inductor y se les pide que asocien a él las tres primeras palabras que se les ocurran en relación con dicho término; b) justificación de las respuestas. El sujeto debe justificar sus respuestas, explicitando las razones por las cuales las han dado y se logra el efecto de clarificar para el sujeto la relación entre el término inductor y las palabras inducidas; c) análisis de la relación entre inductor e inducciones. Se presenta a los sujetos los 28 conectores, tomando la forma de expresiones estándar extraídas del lenguaje corriente, definidos por el modelo para cada una de sus tres respuestas (Guimelli, 2003).

Se han utilizado como términos inductores aquellas palabras que habíamos definido como centrales al evocar al MAG: igualdad, protesta, utopía, diversidad, anticapi- 
talismo y violencia (Cárdenas \& Blanco, 2004) y que coincidieron con las más frecuentemente evocadas en la aplicación piloto de la escala SCB.

\section{Resultados}

El tratamiento de los datos obtenidos por medio de la aplicación del cuestionario de SCB nos entrega un índice o valencia asociado a cada uno de los estímulos inductores (Vt) y que resulta de la división simple del número de asociaciones por el número total de conectores para las tres asociaciones de cada sujeto. Este índice ha sido tratado de tal manera de corregir ciertos problemas asociados a su utilización directa (sobre activación de elementos periféricos o baja activación de aquellos centrales), por lo que se ha realizado el cálculo corregido o índice $\lambda^{1}$ (Guimelli, 2003; Rouquette \& Rateau, 1998) y un índice de incertidumbre o error $(\Delta)^{2}$ que nos otorga unos límites dentro de los cuales oscilaría u elemento central (Guimelli, 2003). Los resultados de los cálculos realizados se pueden observar en la Tabla 1.

Como puede observarse, de las seis palabras con mayor conectividad cuantitativa, hemos podido obtener información que nos autoriza a afirmar que sólo los conceptos de igualdad y protesta pertenecerían al núcleo de la represen- tación social que la muestra con que trabajamos ha elaborado sobre el movimiento Antiglobalización (MAG).

Podemos suponer que aquellos elementos asociados al MAG, y que no se ajustan a los niveles necesarios para incluirlos como elementos centrales según los cálculos realizados, estarían indicando que aún siendo elementos periféricos, logran un alto grado de saliencia (centralidad cuantitativa) que puede explicarse al tratarse de conceptos fuertemente asociados contingentemente a las prácticas del MAG o excesivamente resaltados por los medios de comunicación. De otro modo, de los conceptos presentados (previa selección de ellos teniendo como criterio la importancia cuantitativa que los resultados de otra muestra similar nos entregó) sólo los referidos a la idea de protesta y de igualdad forman parte integrante del núcleo de la representación del MAG y no podría entenderse a dicho movimiento sin ellas.

Sobre las seis palabras utilizadas como estímulo inductor hemos realizado comparaciones sobre sus valencias totales, de modo de precisar si las diferencias son estadísticamente significativas, y precisar dónde concretamente se encuentran esas diferencias. Así, constatamos que existen diferencias entre las valencias totales de las seis formas utilizadas $[\mathrm{F}(79,5)=3.383 ; \mathrm{p}=0.01]$. Por otra parte, consta-

Tabla 1. Valencias Asociadas a los Elementos Centrales y Periféricos.

\begin{tabular}{lcccccc}
\hline & Igualdad & Diversidad & Violencia & Protesta & Anticapitalismo & Utopía \\
\hline Vt & 0.44 & 0.433 & 0.313 & 0.529 & 0.388 & 0.349 \\
$\mathrm{Vp}$ & 0.472 & 0.463 & 0.402 & 0.579 & 0.412 & 0.335 \\
$\mathrm{Va}$ & 0.445 & 0.405 & 0.20 & 0.492 & 0.382 & 0.426 \\
$\Delta$ & 1.405 & 1.145 & 1.549 & 0.917 & 1.232 & 1.191 \\
$\Delta$ & 0.0958 & 0.0965 & 0.1277 & 0.109 & 0.1061 & 0.1426 \\
Medida & $1.045<1.0958$ & $1.145>1.065$ & $1.549>1.1277$ & $0.81<0.917$ & $1.232>1.1061$ & $1.191>1.426$ \\
Diagnóstico & Central & Periférico & Periférico & Central & Periférico & Periférico \\
\hline
\end{tabular}

Tabla 2. Comparación de Inductores sobre Violencias Totales.

\begin{tabular}{lcc}
\hline \multicolumn{1}{c}{ Elementos comparados } & $\mathbf{T}$ & $\mathbf{p}$ \\
\hline $\begin{array}{l}\text { lgualdad (central) } \\
\text { vs Protesta (central) } \\
\begin{array}{l}\text { Protesta (central) } \\
\text { vs Utopía (periférico) }\end{array}\end{array}$ & -1.317 & 0.235 \\
$\begin{array}{l}\text { Protesta (Central) } \\
\text { vs Violencia (periférico) }\end{array}$ & -3.834 & 0.001 \\
$\begin{array}{l}\text { Protesta (Central) vs } \\
\text { Anticapitalismo (periférico) }\end{array}$ & 3.004 & 0.005 \\
\hline
\end{tabular}

tamos que no existen diferencias entre los elementos considerados centrales en el análisis anterior $[\mathrm{T}(25)=-1.217$; $\mathrm{p}=0.235$ ) y que las diferencias se hallarían entre el elemento central Protesta y tres de los estímulos periféricos (ver Tabla 2): violencia, utopía y anticapitalismo.

Ciertamente, era esperable encontrar diferencias entre todos los ítems inductores periféricos respecto de los centrales. Ahora bien, estas discrepancias respecto de nuestras expectativas se pueden deber al bajo número de sujetos que han contestado el cuestionario en algunas de sus modalidades. Creemos que en la medida en que el número de sujetos se incremente, aparecerán dichas diferencias. Por ahora podemos contentarnos con establecido el índice de valencia total como un elemento robusto para distinguir la cen- 
tralidad cualitativa de los diferentes inductores y con conseguir algunos índices estadísticamente significativos entre los elementos centrales y periféricos. También se ha constatado la igualdad de medias entre los dos conceptos centrales.

Resulta interesante evidenciar la gran utilidad del índice lambda y del cálculo del error para discernir entre ítems centrales y periféricos. Esto queda claro al comparar, por ejemplo, la similitud entre las valencias de los conceptos de Igualdad y Diversidad ( 0.44 y 0.433 respectivamente), los que sólo podemos distinguir por medio los procedimientos señalados. Esto vendría a ratificar la idea de que si bien la valencia sería un elemento importante para verificar la centralidad de un elemento, esta por sí misma no basta en tanto puede encubrir el hecho de que un determinado contexto puede resaltar ciertos elementos periféricos y hacerlos aparecer como centrales, y que en otras ocasiones (que no es el caso de nuestro ejemplo) los elementos centrales pueden verse opacados y aparecer con una baja activación.

Aún hay otros datos que queremos comentar y que se refieren a la diferente naturaleza, normativa o funcional, asociada a los elementos nucleares dentro de la RS analizada. Esta naturaleza es puesta en evidencia a través del análisis de las valencias parciales de los esquemas praxis y atribución (Guimelli, 1998; Rateau, 2002). El cálculo de estas valencias parciales nos brinda la posibilidad de acceder a un análisis fino sobre la estructura del núcleo. De este modo, podemos dividir los elementos centrales de una representación en tres tipos diferenciados (Guimelli, 2003): 1) elemento central funcional. La aparición de este tipo de elementos suele tener lugar en sujetos que han llevado a cabo prácticas en relación al objeto de la representación. Los conectores utilizados son de tipo práctico y son activados cuando los sujetos movilizan sus conocimientos o experiencias prácticas al evaluar el objeto de representación. Se espera una alta activación de los operadores de la familia Praxis; 2) elemento central normativo. Suelen aparecer en sujetos que no tienen experiencias concretas en relación al objeto representado y por ello se limitan a realizar juicios normativos derivados de los valores imperantes en el grupo al que pertenece. Aquí se espera una valencia elevada asociada al SCB Atribución; 3) elemento central mixto. Mostrarían valores parejos para los SCB Praxis y Atribución. Los elementos centrales mixtos han demostrado desarrollar un rol más decisivo a la hora de llevar a cabo el reconocimiento y la identificación del objeto.

Los datos obtenidos nos enseñan que la protesta se comporta como un elemento funcional dentro del sistema central de la RS del MAG elaborada por los estudiantes. Es decir, señala que los sujetos poseen algún tipo de implicación directa con el movimiento a nivel de sus prácticas sociales (es probable que incluso algunos de ellos participen activamente del mismo o de algunas de sus activi- dades o actividades de protesta). La igualdad sería un elemento mixto del núcleo de la representación, de manera que es probable que aparezca como el elemento decisivo a la hora de reconocer o identificar la esencia del MAG. Implicaría un reconocimiento y una adhesión a ciertos valores proclamados como bandera de lucha por el MAG y una atribución de estos al mismo, como portador o garante de ellos.

\section{Discusión}

Los datos obtenidos son coincidentes con los resultados que expusiéramos con anterioridad en otro artículo (Cárdenas \& Blanco, 2004), a saber, que los estudiantes elaboran su representación social (RS) sobre el MAGdesde ciertas categorías estables de juicio. Ahora bien, en esa ocasión debíamos concluir que nos faltaban antecedentes para determinar si los elementos semánticos asociados al MAG eran, más allá de su importancia cuantitativa, todos ellos pertenecientes al núcleo de la representación. Es decir, si bien sabíamos cuales eran las dimensiones desde las que se realizaba la selección de los elementos concretos de la representación, nos faltaba aún por determinar cuáles de las asociaciones cualitativamente relevantes y organizadoras del resto de los elementos. Es decir, de entre las palabras con mayor frecuencia, nombradas y utilizadas para describir al MAG y que eran coincidentes con otras aplicaciones que realizamos sobre el mismo instrumento (Protesta, Utopía, Diversidad, Anticapitalismo, Violencia e Igualdad), debíamos decidir cuáles correspondían a elementos centrales y por tanto insustituibles de la RS, y cuáles correspondían a elementos periféricos pero sobre activados.

A partir de la aplicación del cuestionario de SCB hemos podido definir que de entre esas asociaciones sólo dos de ellas mantienen conexiones numerosas y diversificadas, suficientes para ser consideradas elementos cualitativamente centrales. Estaríamos ante un universo consensuado de sentido que tiende a vincular la idea del MAG necesariamente con la protesta y la búsqueda de igualdad. Es decir, no se puede comprender al MAG sin la alusión a su modo de expresión y al horizonte al que apunta su acción. En este sentido, la asociación del movimiento a la izquierda y al término anticapitalismo viene dada por los contenidos históricamente asociados a ella, a saber, la búsqueda de igualdad y la lucha contra las causas percibidas de ésta. Otros conceptos aledaños al de protesta, como lo es violencia, indicarían lo que para ese entonces se consideraba como el resultado más directamente perceptible de las jornadas de protesta Antiglobalización: la represión y las reacciones de violencia que la acompañaban.

En resumen, podemos afirmar que los datos obtenidos por medio del cuestionario de SCB son consistentes con 
los de estudios previos, y que vienen delimitar de mejor manera y a confirmar los resultados a los que llegábamos entonces respecto de definir la RS del movimiento Antiglobalización. Ahora hemos agregado la constatación de los elementos nucleares y hemos descartado una serie de conceptos periféricos pero que se encontraban sobre activados al momento de realizar dicho estudio. Por otra parte, la utilización en este estudio del procedimiento de SCB para confirmar la centralidad de los elementos de una representación contribuye a poner de manifiesto la importancia de utilizar nuevos procedimientos para afinar las técnicas más al uso, mostrando que esta en particular es un instrumento útil y robusto no sólo aportan en sentido de dichas confirmaciones, sino que entrega nuevos elementos mucho más refinados para el análisis de la representación social. Para el caso que analizamos, muestra cómo esta RS se forja desde elementos funcionales que implican la vivencia directa de participación en el movimiento o en algunas de sus actividades, así como elementos de tipo mixto que implicarían que esa participación activa ha sido producto de un proceso reflexivo de atribución de contenidos y de adhesión a valores.

Finalmente, se puede afirmar que es posible un análisis más detallado si se quiere al centrarse en la relación existente entre las palabras que funcionan de estímulo y sus asociaciones por la vía de revisar los conectores asociados a ellas. Es decir, si antes nos podían quedar dudas sobre el sentido o los usos posibles de un término, estas quedan despejadas al centrarse en los operadores utilizados para evocarlas. Obviamente, nos parece que aún queda mucho trabajo por realizar, sobre todo respecto de la necesidad de afinar aún más dichos conectores (que representan operaciones lógicas) y sobre la definición de su número e importancia. En este sentido, nos parece que abundar en esta línea de trabajo puede ser, además de pertinente, enormemente eficaz.

\section{Notas}

$1 \quad \lambda=\mathrm{Vt} /\left(\mathrm{Vp}^{2}+\mathrm{Va}^{2}\right) ;$ donde $\mathrm{Vt}=$ valencia total, $\mathrm{Vp}=$ valencia del $\mathrm{SCB}$ praxis y $\mathrm{Va}=$ valencia del $\mathrm{SCB}$ atribución.

$2 \quad \Delta \lambda=\left[(\mathrm{Vp}-0.5)^{2}+\mathrm{Vp} / 12\right] / 2 \mathrm{Vp}+\left[(\mathrm{Vp}-\mathrm{o.5})^{2}+\mathrm{Vp} / 12\right]$ $\left[(\mathrm{Va}-0.5)^{2}+\mathrm{Va} / 7\right] / 2$.

\section{Referências}

Abric, J-C. (1976). Jeux, conflits et représentations sociales. Thèse de doctorat es lettre, Université de Provence.

Abric, J-C. (1989). L'ètude expérimentale des représentations sociales. In D. Jodelet (Ed), Les représentations sociales (pp. 187203). Paris, PUF.

Abric, J-C. (1994). Pratiques sociales et représentations. Paris: PUF.
Abric, J-C. (2003). Méthodes d'étude des représentations sociales. Editións Érès: Ramonville Saint-Agne.

Cárdenas, M. \& Blanco, A. (2004). Las representaciones sociales del movimiento Antiglobalización. Revista de Psicología Política, $28,27-54$.

Flament, C. \& Rouquette, M-L. (2003). Anatomie des ideés ordinaires. Comment étudier les représentations sociales. Armand Colin/ VUEF, Paris.

Flament, C. (1989): Structure et dynamique des representations socials. In D. Jodelet (Ed.), Les représentations sociales (pp. 204219). Paris: PUF.

Flament, C. (1994). Structure, dynamique et transformation des représentations sociales. In J-C. Abric (Ed.), Practiques sociales et représentations (pp. 37-57). Paris, PUF.

Guimelli, Ch. (1994). Structures et transformations des représentations sociales. Neuchâtel, Delachaux et Niestlé.

Guimelli, Ch. (1995). Valence et structure des représentations sociales. Bulletin de psychologie, Tome XLIX, 422, 58-72.

Guimelli, Ch. (1998): Diferenciation between the central core elements of social representations: normative vs functional elements. Swiss Journal of Psychology, 57, 4, 209-224.

Guimelli, Ch. (2003). Le modèle des schèmes cognitifs de base (SCB). Mèthodes et applications. In J.-C. Abric (Ed.), Méthodes d'étude des représentations sociales (pp. 119-143). Editións Érès: Ramonville Saint-Agne.

Guimelli, Ch. \& Rouquette, M-L. (1992). Contribution du modèle associatif des schèmes cognitifs de base à l'analyse structurale des représentations sociales. Bulletin de psychologie, $X L V, 405$, 196-202

Jodelet, D. (Ed), (1989). Les représentations sociales. Paris, PUF.

Moliner, P. (1989). Validation expérimentale de l'hypothèse du noyau central des représentations sociales. Bulletin de Psychologie, XLI, 759-7622.

Moliner, P. (1992). Représentations sociales Schèmes conditionnels et schèmes normatifs. Bulletin de psychologie, XLV, 405, 325-329.

Moliner, P. (1994). Les methods de repérage et d'identification du noyau des représentations. In Ch. Guimelli (Ed.), Structures et transformation des représentations sociales (pp. 199-232). Neuchâtel, Delachaux et Niestlé.

Moliner, P. (1995). A two-dimensional model of social representations. European Journal of Social Psychology, 25, 27-40.

Moliner, P., Rateau, P.Y. \& Cohen-Scali, V. (2002). Les represéntations sociales. Pratique des études de terrain. Rennes: PUR.

Moscovici, S. (1961). La psychanalyse, son image son public. Paris: PUF.

Rateau, P. (2002): Procédure de substitution et natura des éléments d'une représentation sociale. Cahiers Internationaux de Psychologie Sociale, 54, 62-69.

Rouquette, M-L. (1994). Una classe de modéles pour l'analyse des relations entre cognèmes. In Ch. Guimelli (Ed.), Structures et transformations des représentations sociales (pp. 153-170). Neuchâtel: Delachaux et Niestlé.

Rouquette, M-L. \& Rateau, P. (1998): Introduction à l'étude des representations sociales. Grenoble: PUG.

Verges, P. (1992). L'Evocation de l'argent: Une méthode pour la définition du noyau central d'une représentation. Bulletin de Psychologie, XLV, 405, 203-209. 
Cárdenas, M.; Rodríguez, R. "Utilización del modelo de Esquemas Cognitivos de Base (SCB)..."

Manuel Cárdenas. Psicólogo, Doutor em Psicologia Social pela Universidad Autónoma de Madrid. Atualmente é professor da Escuela de Psicología de la Universidad Católica del Norte Endereço para correspondência: Escuela de Psicología, Universidad Católica del Norte, Av. Angamos 0610, Antofagasta, Chile. jocarde@ucn.cl

Roberto Rodríguez é Psicólogo, Doutor em Psicologia Social pela Universidad Complutense de Madrid.
Utilización del modelo de Esquemas Cognitivos de Base (SCB) para la confirmación del núcleo de una representación social. Análisis del Movimiento Antiglobalización

Manuel Cárdenas y Roberto Rodríguez

Recebido: 24/04/06

$1^{\text {a }}$ revisão: 31/07/06

$2^{\mathrm{a}}$ revisão: 08/09/06

Aceite final: 03/10/06 